\title{
Saúde Mental nas Práticas em Saúde - A Experiência do PROLIG
}

Mental health in the health practice - PROLIG's experience

Salud mental en las prácticas en salud - la experiencia del PROLIG

Samantha Mucci, Fátima Lucchesi, Tatiana Gottlieb Lerman, Daniela Betinassi Parro-Pires, Luciana Geocze, Ligia Bruhn de Souza Aranha \& Mario Alfredo De Marco

Universidade Federal de São Paulo - UNIFESP

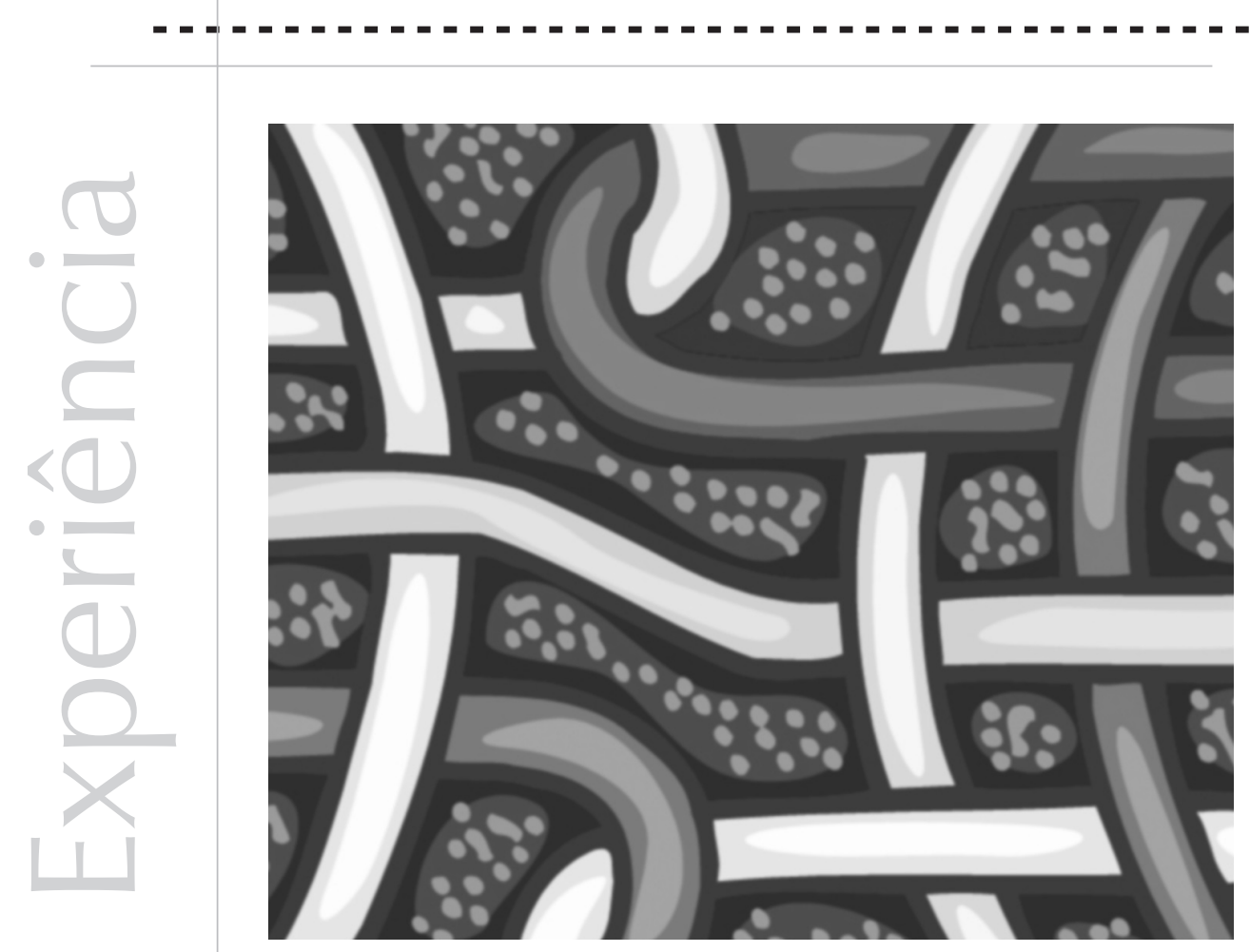


Resumo: O presente trabalho apresenta o relato de experiências do Serviço de Atenção Psicossocial Integrada em Saúde (SAPIS) nos Programas de Ligação (PROLIG). O SAPIS foi implantado em junho de 2002, no Hospital São Paulo (HSP), com o objetivo de promover uma atenção mais ampla às necessidades gerais e específicas do hospital no que diz respeito aos aspectos psicossociais bem como de reorganizar a inclusão de profissionais de saúde mental nas enfermarias e ambulatórios. Esse estudo tem como objetivo descrever a atuação do psicólogo como profissional de ligação em saúde mental no atendimento às demandas específicas de unidades do HSP. A tarefa do profissional de ligação em saúde mental envolve a assistência psicológica ao paciente e seu familiar assim como o desempenho do papel de especialista em saúde mental junto à equipe multiprofissional. Temos encontrado em nossa prática, no entanto, algumas dificuldades para a implementação e a manutenção de algumas propostas. Concluímos que a atuação do psicólogo como profissional de ligação de saúde mental tem promovido mudanças nos cuidados em saúde nessa instituição, porém ainda exige uma profunda reflexão e maior envolvimento da instituição e de políticas de saúde que viabilizem mudanças estruturais para a construção de uma efetiva prática integral e integrada nessa área.

Palavras-chave: Saúde mental. Psicologia da saúde. Interconsulta psiquiátrica.

Abstract: This paper presents the experience of the Psychosocial Service of Integrated Attention in Health (SAPIS) in a consultation-liaison psychiatry program (called PROLIG). The SAPIS was implemented in June 2002 at the São Paulo Hospital (HSP) to promote a broad attention to general and specific hospital psychosocial needs and also to reorganize the inclusion of mental health professionals in ambulatories and wards. This paper aims at describing the role of psychologists as mental health liaison professionals attending specific demands at these units of HSP. Their task involves providing psychological assistance to the patient and his family, as well as being a mental health specialist to the multi-professional team. Nevertheless, some difficulties have been found to implement and maintain these proposals. Concluding, psychologists as mental health liaison professionals promote changes in the health care offered by this institution; however, a deeper reflection and engagement of this institution as well as an implementation of health care policies are demanded to enable structural changes for the construction of an integral and integrated effective practice.

Keywords: Mental Health. Health Psychology. Consultation-Liaison Psychiatry.

Resumen: El presente trabajo presenta el relato de experiencias del Servicio de Atención Psicosocial Integrado en Salud (SAPIS) en los Programas de Conexión (PROLIG). El SAPIS fue implantado en junio de 2002, en el Hospital Sao Paulo (HSP), con el objetivo de promover una atención más amplia a las necesidades generales y específicas del hospital en lo que se refiere a los aspectos psicosociales así como de reorganizar la inclusión de profesionales de salud mental en las enfermerías y ambulatorios. Este estudio tiene como objetivo describir la actuación del psicólogo como profesional de conexión en salud mental en el servicio a las demandas específicas de unidades del HSP. La tarea del profesional de conexión en salud mental implica la asistencia psicológica al paciente y su familiar así como el desempeño del papel de especialista en salud mental junto al equipo multiprofesional. Hemos encontrado en nuestra práctica, sin embargo, algunas dificultades para la implementación y el mantenimiento de algunas propuestas. Concluimos que la actuación del psicólogo como profesional de conexión de salud mental ha promovido cambios en los cuidados en salud en esa institución, sin embargo aún exige una profunda ponderación y mayor implicación de la institución y de políticas de salud que hagan viables los cambios estructurales para la construcción de una efectiva práctica integral e integrada en esa área.

Palabras-clave: Salud mental. Psicología de la salud. Interconsulta psiquiátrica.

\section{Saúde mental nas práticas em saúde: premissas e perspectivas}

No quadro da crescente especialização, o papel do psicólogo como profissional de ligação em saúde mental tem incorporado características peculiares. A especialização é fato e necessidade, tendo em vista a evolução e o crescimento exponencial dos conhecimentos. É importante fazer distinção entre uma especialização integrada, que se focaliza num campo de conhecimento, sem 


\begin{abstract}
É importante fazer distinção entre uma especialização integrada, que se focaliza num campo de

conhecimento, sem perder de vista a articulação com os demais aspectos e dimensões do ser e dos cuidados, e um "loteamento rígido", que poderia trazer a perda da integralidade $e$ efetividade
\end{abstract}

(De Marco, 2003). perder de vista a articulação com os demais aspectos e dimensões do ser e dos cuidados, e um "loteamento rígido", que poderia trazer a perda de integralidade e efetividade (De Marco, 2003).

Nesse cenário, nossas atribuições enquanto profissionais de saúde mental têm percorrido uma trajetória que nos colocam, de um lado, como mais um especialista a ocupar-se de sua área específica e, de outro, como um dos articuladores privilegiados do processo de integração e integralidade dos cuidados. Algumas características de nosso campo de interesse nos qualificam como um dos elementos de referência na gestão do interesse e das ações visando à integralidade dos cuidados: a saúde mental se dedica ao estudo das perturbações do contato com a realidade, e envolve um compromisso com uma epistemologia da observação. O transtorno mental, independentemente de questões etiológicas, acarreta sempre perturbação do contato com a realidade, e a nossa formação está (ou pelo menos deveria estar) voltada para a evolução de nossas capacidades para reconhecer e procurar os equacionamentos possíveis para essas distorções. O "loteamento rígido" dos cuidados em saúde decorre e/ou acarreta distorção da observação e do contato com a realidade que repercute na qualidade do vínculo e da comunicação, e, em conseqüência, nos cuidados e na evolução. Esse é um dos fatores que motiva e determina nosso engajamento para além da atuação na área específica da especialização, no que podemos denominar de "terapia da tarefa" assistencial em seus diferentes níveis (ensino, assistência e pesquisa) e contextos (relacional, ambiental e institucional), contribuindo assim para o processo de integração e integralidade dos cuidados, que envolve uma série de evoluções e transformações, entre as quais: deslocamento do foco da doença como evento para o adoecer como processo, incorporação às ações das perspectivas de abrangência e continuidade dos cuidados, aumento da abrangência e integração dos diferentes cenários, adequação dos programas de ensino, educação continuada e promoção de cuidados de saúde e qualidade de vida para estudantes e trabalhadores da área de saúde, capacitação e educação continuada de pacientes e familiares e promoção e ampliação do trabalho em equipe multiprofissional (De Marco, Citero, \& Nogueira-Martins, 2007).

A contribuição da saúde mental nesse processo vem se consolidando e ampliando, e um cuidado que se deve ter é procurar manter esse movimento vivo, a fim de não permitir que o campo da saúde mental perca essa sua característica e se restrinja ao papel de mais uma especialidade a lotear o campo da saúde.

\section{O Hospital São Paulo (HSP)}

O HSP foi fundado em 1937, com um pavilhão de 100 leitos, no bairro da Vila Clementino, na capital de São Paulo, para se tornar o cenário de ensino prático dos alunos da Escola Paulista de Medicina. Atualmente, é campo de ensino e treinamento da Universidade Federal de São Paulo (UNIFESP), e colabora para a formação e a capacitação de profissionais de saúde. Possui 743 leitos de internação, sendo 651 leitos para adultos e 92 para a pediatria, e atende diariamente mais de 4.500 pacientes ambulatoriais e 1.200 no pronto-socorro/pronto-atendimento. Tem como missão a prestação de assistência à saúde à população brasileira através do Sistema Único de Saúde (SUS). 


\section{A experiência do SAPIS}

A interconsulta em saúde mental: foi criada em 1977, no Departamento de Psiquiatria da UNIFESP, campus São Paulo, inspirada na proposta de Ferrari e Luchina (1971), cujo modelo tem como base fundamental para a intervenção no campo a perspectiva psicanalítica. Construiu, gradativamente, um perfil próprio, e apresenta, neste momento de sua evolução, as seguintes características básicas: intervenção orientada a partir do campo interacional, estruturada a partir da observação do campo que se configura na interação entre a equipe de saúde (incluindo o ambiente hospitalar e o plano institucional) e o grupo sob atenção (que envolve paciente, familiares e a rede social); atendimento especializado integrado à perspectiva do campo, sempre considerado em função da perspectiva mais ampla, mesmo nos casos em que, aparentemente, apenas uma intervenção específica é requisitada e/ou pareça necessária; educação permanente dos profissionais, estudantes, pacientes e familiares, sensibilizando-os para os fenômenos da vida mental e emocional e da dimensão social presente e atuante nas situações.

Desenvolvimentos: A partir de 1999, surgiu um interesse mais consistente no sentido de uma integração e de uma atuação mais ampla em saúde mental no HSP. Para efetivar a integração, foi decidida a implantação de um serviço visando a promover uma atenção mais ampla às necessidades gerais e específicas do hospital e seus setores bem como a reorganizar a inclusão de profissionais de saúde mental nas enfermarias e ambulatórios, com a definiçãa de critérios de inserção e de capacitação desses profissionais. O serviço se articula em duas frentes de organização:
A-programas centralizados: referem-se às medidas gerais para a instituição como um todo, e são dirigidas tanto aos pacientes e familiares quanto à equipe de saúde e administrativa.

\section{B-programas descentralizados: Programas de Ligação em Saúde Mental (PROLIG)} São ações e programas elaborados de acordo com as demandas, específicos para setores, enfermarias ou ambulatórios, que visam a desenvolver programas específicos que atendam as necessidades peculiares de cada serviço, com prioridades de oferta e de demanda.

Atualmente, a equipe de profissionais de ligação é constituída por seis psicólogos com especialização em saúde mental pela UNIFESP, distribuídos em unidades de internação e ambulatoriais do HSP.

\section{Implementação e Desenvolvimento do PROLIG}

A partir de critério de demanda e otimização dos recursos, o PROLIG implantou o programa, desde 2002, no Serviço de Transplante de Fígado, nas enfermarias de clínica médica e nas enfermarias cirúrgicas. Em 2003, o programa foi implantado na Enfermaria de Hematologia e Transplante de Medula Óssea e na Enfermaria de Doenças Infecto-Parasitárias (DIPA), e, em 2004, nas unidades de terapia intensiva (UTI).

\section{Aspectos gerais do PROLIG}

Características emocionais mais comuns observadas nos pacientes e/ou familiares

Podemos observar algumas características psíquicas comuns aos pacientes/familiares 
internados ou acompanhados pelos profissionais de ligação, como ansiedade pela espera (demora dos resultados de exames e condutas das interconsultas), ansiedade frente aos exames e procedimentos (exames de sangue diários, ultrassom, ressonância magnética, entre outros), medo de terem uma doença grave, contagiosa, incapacitante e incurável, associado a fantasias e crenças quanto ao diagnóstico, como a dificuldade de adaptação à nova situação do organismo, o medo da dor e da morte, dificuldade de adesão ao tratamento, perda da autonomia e necessidade de apoio dos familiares, ansiedade e tensão decorrentes do tempo de internação, expectativas quanto ao resultado do tratamento, conflito pelo afastamento do trabalho ou escola devido às limitações físicas ou internação, dificuldade econômica (diminuição da renda familiar e aumento dos gastos), sentimentos de culpa por estar causando transtorno e preocupação à família e necessidade de apoio e proximidade dos familiares.

Também é comum observar, após o diagnóstico ou indicação de procedimento cirúrgico, a presença de reações defensivas, como a recusa da existência da doença, a negação do sentimento gerado em decorrência do diagnóstico, a projeção da raiva na equipe, o sentimento de revolta por estar doente e a regressão (atitude infantilizada frente aos sentimentos de desamparo). Temos ainda insegurança, dificuldades relacionadas a conversar ou vivenciar a proximidade real da morte bem como tristeza, raiva e incompreensão dos motivos de ter ficado doente.

Características da equipe assistencial (médicos, enfermeiros e auxiliares de enfermagem)

Em relação à equipe, observamos algumas dificuldades no que se refere ao contato com o paciente e/ou familiares: medo da reação do paciente diante do diagnóstico dado pelo médico (principalmente nos casos de conduta reservada), dificuldade em lidar com os familiares (o residente não conversa com o familiar, e os profissionais não hesitam em dar a notícia de um prognóstico muito ruim ou fechado para família e paciente, mas o contato geralmente é breve, para evitar lidar com os sentimentos despertados. Isso acaba aumentando a dificuldade dos familiares de escutar aquilo que lhes é dito num primeiro momento).

A equipe apresenta, com alguma freqüência, dificuldades em lidar com pacientes que negam a doença, recusam o tratamento ou tornamse hostis. Essas dificuldades estão ligadas à comunicação de más notícias, dificuldade de lidar com os aspectos emocionais do paciente e hesitação em dar a notícia de um prognóstico muito ruim ou fechado para família e paciente.

\section{Dinâmica do PROLIG}

As ações são orientadas tanto por demanda quanto por projetos de intervenção sistematizados. Os atendimentos por demanda são realizados segundo solicitação da equipe assistencial, do próprio paciente, de seus familiares ou a partir de detecção pelo próprio profissional de ligação, conforme observação da dinâmica nas enfermarias, na Unidade de Terapia Intensiva ou no Serviço de Transplante de Fígado. A solicitação da equipe assistencial ao profissional de ligação geralmente é feita para auxiliá-la no manejo de pacientes que estão prestes a receber um diagnóstico difícil, para orientação a pacientes com dificuldade de aderir ao tratamento, dúvidas quanto a diagnósticos psiquiátricos (transtorno de ajustamento ao diagnóstico, ao tratamento ou à recidiva da doença, quadros ansiosos 
ou depressivos) e dificuldades de lidar com aspectos emocionais do paciente/família. Após o encaminhamento ou solicitação de atendimento, o profissional de ligação realiza os seguintes procedimentos:

Com o paciente: avaliação psicodiagnóstica individual e diagnóstico situacional, incluindo dados sociodemográficos, suporte social, identificação de hábitos, nível de compreensão com relação à doença e ao tratamento, capacidade de enfrentamento (coping) e adesão ao tratamento, avaliação em saúde mental (exame psíquico, tratamento atual ou prévio em saúde mental, psicopatologias pré-existentes ou em risco de desenvolvimento) e avaliação dos riscos e benefícios dos tratamentos em curso ou preconizados. Realizada a avaliação do paciente, são sugeridas condutas (atendimento psicoterápico individual, atendimento familiar e encaminhamentos, terapia ocupacional, avaliação psiquiátrica, assistente social) de acordo com a necessidade do paciente. Para avaliação e acompanhamento psiquiátrico, é acionada a interconsulta em saúde mental.

Com o familiar: avaliação de sua compreensão da doença e sensibilização para sua aceitação e compreensão da necessidade dos cuidados a serem dispensados ao doente inclusive após a alta hospitalar, orientações visando a facilitar seu relacionamento com o paciente e a comunicação com a equipe, obtenção de informações sobre a história de vida e o comportamento do paciente antes da internação/adoecimento, tendo em vista compreender como o mesmo lida com as situações de crise.

Com a equipe de saúde: acolhimento do pedido da equipe, com algumas orientações sobre o manejo do paciente. Assim que a avaliação do paciente é realizada, o profissional solicitante é procurado para discutir o caso, tendo sempre em foco também as questões emocionais do profissional envolvidas na demanda.

Por seu turno, os projetos de intervenção sistematizados incluem as diferentes ações programadas para favorecer um atendimento integral e integrado e a ampliação de uma participação efetiva de todos os envolvidos no processo (pacientes, familiares, equipe, comunidade).

Os profissionais de ligação também realizam as seguintes intervenções sistematizadas: trocas de informações cotidianas sobre os pacientes com a equipe médica e de enfermagem, participação em visitas médicas (com a meta de contribuir para uma educação continuada em relação ao profissional de saúde não especializado em saúde mental), participação em reuniões ou discussões clínicas, nas quais se trocam informações sobre os pacientes/família, procurando-se facilitar a compreensão da dinâmica das relações entre os membros da equipe, entre a equipe e o paciente/família e o reflexo dessa dinâmica nas condutas e procedimentos. As reuniões e discussões clínicas também representam uma oportunidade para tentar orientar a equipe sobre as condutas que possam facilitar a comunicação com o paciente, a adesão ao tratamento e a diminuição do risco de desenvolvimento de distúrbios emocionais. Com a troca de informações entre os profissionais e uma ampliação da compreensão dos casos clínicos, procura-se favorecer a tomada de decisões em equipe.

\section{Características específicas dos serviços}

Serviço de Transplante de Fígado: foi criado em 1998, tendo o primeiro transplante sido 
Outros estudos mostram que $20 \%$ dos candidatos a transplante de fígado têm, concomitantemente, transtornos de ajustamento e 4,5\% tem depressão maior; $9 \%$ preenchem critérios para abuso ou dependência de álcool e $2 \%$ para abuso de outras substâncias

(Trzepacz, Brenner, \& Van Thiel, 1989) realizado em março de 2000 . Já foram realizados 75 transplantes de adultos e 15 de crianças. Atualmente, há aproximadamente 400 pacientes adultos e 50 crianças na lista à espera de transplante.

Nesse serviço, há predominância de quadros de ansiedade, depressão e transtornos de ajustamento nos pacientes. Durante a internação, no pós-transplante recente, a maioria dos pacientes apresenta quadros de delirium. Na literatura, encontram-se resultados semelhantes: taxas de depressão com 20 a 45\% de prevalência em pacientes transplantados cardíacos (Frasur-Smith, Lesperance, \& Talajic, 1993; Schleifer et al., 1989). Em serviço de transplante de pulmão, até $50 \%$ dos pacientes apresentam história de transtorno psiquiátrico (Craven, 1990) Outros estudos mostram que $20 \%$ dos candidatos a transplante de fígado têm, concomitantemente, transtornos de ajustamento e 4,5\% tem depressão maior; $9 \%$ preenchem critérios para abuso ou dependência de álcool e 2\% para abuso de outras substâncias (Trzepacz, Brenner, \& Van Thiel, 1989) Em pacientes submetidos à diálise, depressão maior tem sido descrita em 5 a 22\% (Lowry, 1979). Rundell (1997) demonstra ainda que pacientes em serviços de transplante diferem daqueles vistos em hospitais gerais e são mais propensos a ter sintomas psiquiátricos.

Os pacientes no pré-transplante apresentam um prejuízo de qualidade de vida decorrente da gravidade da doença hepática e dos sintomas como ascite (barriga d'água), icterícia (amarelão) e encefalopatia (quadro de confusão mental secundário à hepatopatia crônica), que produzem limitações para realizar atividades diárias e auto-cuidado, limitações em manter uma atividade social e sintomas de ansiedade relacionados à possibilidade de realização do transplante.
As atividades sistematizadas desenvolvidas pelo profissional de ligação no serviço de transplante de fígado são: protocolo de avaliação psicossocial para candidatos a transplante intervivos de fígado, brinquedoteca, grupo psicopedagógico para pacientes pré-transplante e familiares, grupos de sala de espera com pacientes/familiares pré e pós-transplantes e grupo de atividades com pacientes pós-transplantes.

\section{Enfermaria de Hematologia e Transplante de Medula Óssea (TMO): com 14 leitos, 2} deles destinados aos pacientes que aguardam transplante ou a familiares que farão a doação de medula. No mesmo espaço, separado por uma porta, está a Unidade de Transplante de Medula Óssea, com cinco leitos.

Muitos pacientes passam por várias outras clínicas antes de chegar à hematologia. Leucemias, linfomas e mieloma múltiplo são os diagnósticos mais comuns dos pacientes internados na unidade. Não há uma faixa etária prevalente entre os pacientes internados, e podem ser encontrados desde jovens adultos a idosos, sem diferenciação com relação ao gênero. Na maioria dos casos, os pacientes já sabem o diagnóstico, alguns acabaram de recebê-lo e outros já fazem tratamento há algum tempo. É comum a presença de pacientes que estão em tratamento ambulatorial há um ano ou mais. O período de internação é bastante variável - desde alguns dias até alguns meses. Re-internações são freqüentes, já que esses tratamentos são de longa duração.

Pacientes que vêm de outros Estados enfrentam maiores dificuldades devido à distância da família e do lar. É acentuado o número de óbitos durante a internação, tanto em decorrência da própria doença como por reações e complicações do uso dos quimioterápicos. 
Os pacientes passam por períodos em que o isolamento e o repouso absoluto são necessários pelo fato de se encontrarem em fase de aplasia da medula (falência da medula óssea, órgão responsável pela produção do sangue, pela produção de células sangüíneas) ou plaquetopenia (diminuição do número de plaquetas no sangue que pode diminuir o potencial de coagulação sanguínea), isto é, os pacientes ficam mais vulneráveis a outras doenças, tais como infecções por bactérias ou vírus, por exemplo, ou ainda a sofrerem sangramentos nasais, intestinais ou em qualquer parte do corpo, sendo necessários, dessa maneira, cuidados especiais. A iminência do transplante de medula é um fator de muito estresse para os pacientes, uma vez que sabem que é um processo difícil de enfrentar devido às reações provocadas pela quimioterapia, pelo temor do aprisionamento e por saberem da necessidade de ficarem restritos ao quarto do TMO, além do medo de não haver resultados positivos.

Pacientes com recidiva da doença merecem atenção ainda maior, uma vez que sentimentos intensos de frustração, raiva, medo, culpa, tristeza e desesperança podem ressurgir de forma mais intensa. O paciente sabe o que o espera, pois já conhece o tratamento, e esse sentimento é acompanhado pelo medo e pela insegurança de um novo insucesso ou do fim de suas chances de cura.

Furlanetto, Del Moral, Gonçalves, Rodrigues e Jacomino (2006) encontraram sintomas depressivos significativos em cerca de $1 / 3$ a $1 / 4$ dos pacientes internados com doenças hematológicas. Os sintomas que melhor os discriminaram foram: sensação de fracasso, anedonia, culpa e fadiga.

A tarefa contínua de cuidar desses pacientes acarreta grande carga emocional. Ocorrem, com freqüência, pedidos de licença médica ou de transferência por parte da equipe de enfermagem, o que dá uma idéia do desgaste intenso vivenciado nessa unidade, além das dificuldades decorrentes das inadequações das políticas públicas de saúde. Há também problemas no número de funcionários da equipe de enfermagem, o que dificulta o cumprimento das exigências da rotina das unidades.

Os residentes mantêm outras atividades em conjunto com sua passagem pela enfermaria e pelo TMO, o que resulta em pouco tempo disponível para lidar com a demanda das unidades, com os pacientes graves e delicados como são os da hematologia e TMO. A equipe de saúde, principalmente a médica, apresenta dificuldades de acompanhamento e comunicação com os pacientes que sofrem intensamente. Quintana, Cecim e Henn (2002, p. 205) sugerem que "essa resistência em comunicar o estado do paciente é diretamente proporcional à gravidade da condição deste".

Enfermaria de Clínica Médica: é composta pela unidade feminina, masculina e a UTI. São internados pacientes para investigação clínica, realização de diagnósticos e tratamento de quadros clínicos de pacientes provenientes do PS do hospital, do ambulatório de clínica médica e por encaminhamento de outras especialidades. Apresentam algumas características particulares por serem enfermarias voltadas principalmente para a investigação diagnóstica, sendo freqüente a demora para o fechamento desse diagnóstico.

A quebra dos vínculos pela internação prolongada e a necessidade de afastamento do trabalho ou até mesmo da família (principalmente em pacientes de outros Estados) são freqüentes e contribuem para 
É difícil precisar com segurança a proporção de pacientes acometidos por transtornos de humor crônicos e diferenciá-los dos agudos, uma vez que a depressão e a ansiedade podem estar relacionadas com a gravidade da doença física ou serem quadros independentes

(Botega et al., 1995; Cavanaugh, Furlanetto, Creech, \& Powell, 2001). a alteração dos estados emocionais. Tais dificuldades estão relacionadas à evolução e ao prognóstico da doença e às limitações físicas e psicossociais (por ex., limitações financeiras, quando o paciente é o provedor da família). Os quadros psiquiátricos mais freqüentes são: transtorno de ansiedade generalizada, transtorno de ajustamento, transtornos do humor com episódios depressivos, confusão mental ou delirium. A mediana da prevalência de transtornos mentais encontrados na clínica geral é de cerca de 25\% dos casos; os transtornos mais freqüentes são: transtornos de ansiedade, depressivos e somatoformes (Mari \& Jorge, 1997). Na literatura, em estudos com pacientes internados em enfermarias clínicas, a prevalência para depressão foi entre 7 e 20\% (Martin, Soler, \& Picart, 2003); 27\% (Creed et al., 2002); 33\% (Botega, Bio, Zomignani, Garcia Jr, \& Pereira, 1995) e 26\% (Cigognini \& Furnaletto, 2006). Em relação aos sintomas de ansiedade, a prevalência foi entre 6 a 17\% (Martin et al., 2003) e 20,5\% (Botega et al., 1995).

É difícil precisar com segurança a proporção de pacientes acometidos por transtornos de humor crônicos e diferenciá-los dos agudos, uma vez que a depressão e a ansiedade podem estar relacionadas com a gravidade da doença física ou serem quadros independentes (Botega et al., 1995; Cavanaugh, Furlanetto, Creech, \& Powell, 2001). Os residentes são orientados quanto à diferença entre o quadro orgânico de delirium e um transtorno psiquiátrico pelos próprios preceptores da clínica, e recebem instruções quanto aos exames necessários para um diagnóstico diferencial, enquanto o diagnóstico para transtorno de depressão e ansiedade, algumas vezes, não é tão prontamente realizado.
A equipe médica, em algumas ocasiões, apresenta dificuldade em lidar com a demora do diagnóstico, sente-se impotente e, em conseqüência, passa a não explicar ao paciente tudo o que está ocorrendo. Pode-se compreender tal comportamento como uma forma de não lidar adequadamente com a sensação de impotência. A equipe pode se sentir ameaçada pelos questionamentos feitos pelos pacientes e familiares e entender as perguntas como uma descrença ou dúvida em relação ao seu trabalho.

A atividade sistematizada desenvolvida pelo profissional de ligação nas enfermarias de clínica médica é a capacitação em saúde mental para a equipe assistencial.

\section{Enfermaria de Doenças Infecciosas e} Parasitárias (DIPA): possui18 leitos, sendo 2 de terapia intensiva e 8 de unidade de internação com pressão negativa (isolamento). Geralmente, a demanda da equipe ao profissional de ligação é de orientação a pacientes com dificuldade de aderir ao tratamento anti-retroviral, auxílio para manejo de pacientes prestes a receber o diagnóstico de AIDS, dúvidas quanto a diagnósticos psiquiátricos e dificuldades com o paciente. Podemos observar algumas características psíquicas específicas dos pacientes portadores do vírus HIV que corroboram os resultados de alguns estudos (Bader et al., 2006; Brousseau, 2007; Sarazua, Mas, \& San Gregório, 2005; Siegel \& Schrimshaw, 2005), que foram a dificuldade de adaptação à nova situação, a sensação de morte iminente, a dificuldade em aceitar procedimentos e as dietas necessárias para o restabelecimento da saúde, além das dúvidas a respeito da medicação e o medo de ser discriminado pela equipe e pelos outros pacientes.

Unidade de Terapia Intensiva: a UTI Geral tem 14 leitos, sendo 4 isolamentos e 
2 reservados para pacientes neurológicos. Recebe pacientes de diversas especialidades, exceto das enfermarias de cardiologia e pediatria. O ambiente físico é pouco acolhedor, e gera sentimentos de ameaça tanto a pacientes quanto a familiares.

Com alguma freqüência, o paciente é mantido em estado de sedação, e, durante o período de recuperação progressiva da consciência, depara-se com uma série de características do ambiente da UTI (Botega \& Smaira, 2006). Existe um constante fluxo de profissionais e estudantes no ambiente e em contato com os pacientes (o quadro de profissionais fixos da unidade é extenso devido à gravidade e à necessidade de cuidados dos pacientes, além dos serviços de interconsulta). Tal situação se reflete nos pacientes e familiares e desperta reações tanto positivas (sentimentos de estarem sendo cuidados e de terem acesso ao melhor em termos de diagnóstico e tratamento) como negativas (falta de privacidade, dor provocada pelos procedimentos invasivos, falha na comunicação entre paciente e médico e discussões diagnósticas e prognósticas feitas pela equipe, em geral, à beira do leito, sem levar em conta a capacidade cognitiva do paciente). Nesse contexto, são notadas as reações do paciente quando este consegue se comunicar ou expressar de alguma forma seu desconforto, angústia ou medo. Às vezes, quem está mais próximo ao paciente, um auxiliar de enfermagem, enfermeiro ou residente, se torna uma pessoa de referência para o paciente ou para a família, e consegue dar algum tipo de acolhimento, mas, em alguns casos, se isso não ocorrer, o paciente ou o familiar pode silenciar ou passar a questionar demasiadamente aquilo que tem sido feito em termos de conduta para o paciente.
Em relação à equipe, observamos algumas dificuldades no contato com o paciente e/ ou familiares: medo da reação do paciente diante do diagnóstico dado pelo médico (principalmente nos casos de conduta reservada), dificuldade em lidar com os familiares (o residente não conversa com o familiar, os profissionais não hesitam em dar a notícia de um prognóstico muito ruim ou fechado para família e paciente, mas evitam lidar com os sentimentos despertados. Isto muitas vezes pode impactar na forma como a família escuta e/ou entende das informações dadas.

Enfermarias cirúrgicas: o SAPIS iniciou a implantação do Programa de Ligação nas enfermarias cirúrgicas pela gastrocirurgia. Esta é uma das maiores do Hospital, com 33 leitos mistos, quase sempre ocupados. A rotatividade de pacientes é bastante alta, e, durante a internação, espera-se que o paciente realize exames complementares, seja preparado para a cirurgia e receba os cuidados para uma boa recuperação clínica. Reuniões para discussão de casos e condutas entre as equipes são realizadas diariamente. Devido à alta rotatividade e à inconstância dos residentes na enfermaria (os procedimentos no centro cirúrgico exigem a presença da equipe médica), o contato com os residentes e os cuidados com os pacientes se tornaram tarefas difíceis. O trabalho não apresentava uma integração entre o profissional de ligação e a equipe médica e a de enfermagem dessa unidade, o que nos levou a uma mudança tática: revisão da abordagem e cuidado desses aspectos psicossociais, buscando-se otimizar a utilização dos recursos e as estratégias de atuação para a área cirúrgica do hospital. A idéia que se apresentou como mais viável para a otimização das nossas ações foi a de desenvolver um protocolo que pudesse proporcionar a detecção e a atenção aos cuidados perioperatórios. 
Para desenvolver esse novo projeto, durante um período de um ano e meio, realizamos a revisão da literatura sobre os cuidados psicossociais envolvidos no período perioperatório, coletamos dados a respeito do funcionamento dessas enfermarias e dos aspectos psicossociais dos pacientes internados, ocasião em que detectamos um número expressivo de pacientes com complicações no âmbito psicossocial como: sintomatologia psiquiátrica presente, suporte social insatisfatório e desinformação a respeito da doença e do momento perioperatório. Esses aspectos se caracterizavam como pontos críticos a serem cuidados durante a internação. Nesse período, também foram realizadas reuniões com a superintendência do HSP e as diretorias das disciplinas cirúrgicas, com o intuito de detectar a necessidade de um cuidado integrado pelos chefes das disciplinas assim como de unir esforços para a implantação do protocolo de forma unificada.

Definidos os focos de nossa atuação, desenhamos um modelo de protocolo de avaliação psicossocial que passaria a fazer parte da rotina das enfermarias, a fim de serem avaliados pontos como grau de compreensão do paciente a respeito da doença, cirurgia, padrão de adesão a tratamentos, suporte social, hábitos e saúde mental.

A ficha de avaliação psicossocial seria aplicada em cada paciente que se submetesse à cirurgia (eletiva ou de emergência), e, a partir do levantamento desses dados, a equipe da enfermaria seria orientada a realizar as condutas necessárias para um atendimento mais integral. Essas condutas estariam explicitadas ao lado de cada resposta do paciente (fácil visualização) para orientar a equipe sobre as condutas necessárias, como, por exemplo, quando encaminhar o paciente para avaliação psiquiátrica ou quando reorientá-lo a respeito de suas condições de saúde, ou, ainda, quando contar com a assistente social para prevenir sobre baixa adesão ao tratamento.

Com o protocolo estruturado, iniciamos sua implantação nas enfermarias de gastrocirurgia e ortopedia do HSP, com o objetivo de realizar um estudo-piloto visando a avaliar a sua aplicabilidade, a compreensão pelos pacientes e a aceitação da equipe, para depois fazermos os ajustes necessários e futuramente estendermos sua implantação às outras enfermarias cirúrgicas do HSP.

\section{Avaliação e Perspectivas}

Através de nossa atuação, foi possível verificar a importância de o psicólogo como profissional de ligação em saúde mental manter-se atento às premissas que enunciamos e não ser simplesmente mais um profissional a "lotear" o cuidado ao paciente. Nossa tarefa como profissional de ligação em saúde mental dentro de uma instituição está voltada tanto para prestar assistência psicológica ao paciente e seu familiar como para desempenhar o papel de especialista em saúde mental junto à equipe multiprofissional, instrumentalizando-a para que ela possa lidar com os aspectos psicológicos relacionados ao adoecer, implantar protocolos de ações específicas e desenvolver estratégias de intervenção.

Nesse papel ampliado, além da execução de cuidados específicos, nosso posicionamento tem permitido observar características e distorções do campo e das relações que interferem na consecução de uma atenção integral e integrada, constituindo-se em importantes focos para a nossa intervenção. É importante, entretanto, ressaltar que as 
propostas de intervenção precisam levar em conta as dificuldades e as resistências que se interpõem para sua implementação e manutenção. A nossa proposta de desenvolver um trabalho interdisciplinar amplo e efetivo nas enfermarias do HSP esbarra, por exemplo, na alta rotatividade da equipe de médicos residentes responsáveis pela assistência (rotatividade mensal) e pelas outras atividades de ensino e atendimentos ambulatoriais e/ ou cirúrgicos durante o mesmo período. Essas características da residência médica dificultam o exercício de um cuidado integral ao paciente/familiar por parte do médico e fazem com que ele disponha de pouco tempo e pouco interesse em trocar informações com o profissional de ligação.

Podemos perceber também que, apesar de as equipes médicas conhecerem a importância da participação da família no tratamento dos pacientes, o observado nas enfermarias é que essa fica, muitas vezes em segundo plano - outras atividades dos médicos residentes acontecem no mesmo horário de visita dos familiares -, o que acarreta dificuldade de comunicação, de esclarecimento de condutas, de tratamento e prognóstico. No caso de uma família mais ativa, a busca de esclarecimentos decorre de sua própria iniciativa, porém essa não é a realidade mais comum em um hospital público; podemos verificar também, como, de maneira geral, troca e comunicação eficiente da equipe médica e enfermagem ficam dependentes das características pessoais de tais profissionais. Apesar da existência da prescrição impressa, há problemas no repasse de informações para a equipe de enfermagem, como, por exemplo, mudanças de conduta em geral, comunicação de diagnósticos graves e planejamento da alta hospitalar.
Outra dificuldade que encontramos está relacionada à baixa remuneração e à sobrecarga de trabalho da equipe de enfermagem. Notamos que, em algumas unidades, a carga emocional é muito intensa, e que o estresse gerado pela tarefa (lidar com pacientes graves, impotência e morte) provoca sentimentos de menos valia, desânimo, tristeza e sensação de que o trabalho não traz resultados. Como conseqüência, e a fim de evitar a frustração e os sentimentos penosos, a equipe passa a não refletir sobre as dificuldades e as repercussões em sua saúde mental.

Para enfrentar essas dificuldades, temos tentado adaptar a inserção do PROLIG nas diferentes enfermarias visando a otimizar o aproveitamento dos nossos recursos. É o caso, por exemplo, das enfermarias cirúrgicas do HSP, nas quais estamos procurando desenvolver um cuidado integral e integrado através da implantação do protocolo de avaliação psicossocial. Pudemos constatar que o protocolo é de fácil compreensão pelos pacientes, sua aplicação é fácil e rápida, e colabora para que o médico ou a equipe de enfermagem detecte questões psicossociais, além de orientá-los sobre o manejo de situações que exijam maior atenção e um cuidado ampliado. Observamos também que a implantação do protocolo deverá ser sempre precedida de um estudo-piloto em cada enfermaria onde seja implantado, com a possibilidade de se fazer adaptações específicas de acordo com a necessidade de cada uma delas.

Diante desse quadro complexo, podemos concluir que a tarefa do psicólogo como profissional de ligação em saúde mental no HSP tem contribuído para promover mudanças nos cuidados em saúde nessa instituição, porém ainda exige maior reflexão por parte do profissional, o envolvimento da instituição e políticas de saúde que 
viabilizem mudanças estruturais para que se possa construir uma efetiva prática integral e integrada.

\section{Considerações finais}

Para finalizar, gostaríamos de fazer uma consideração importante quanto à questão da inserção do psicólogo como profissional de ligação em saúde mental em instituições hospitalares. Existe uma discussão sobre qual é o melhor modelo: o profissional de saúde mental formalmente ligado ao serviço onde exerce sua atividade ou ligado a um serviço/ departamento que estabeleça as políticas de atuação e desenvolvimento da área?
Para o modelo que estamos propondo, nossa convicção é a importância de os profissionais de saúde mental estarem ligados a um mesmo serviço ou departamento que estabeleça as políticas de atuação e desenvolvimento, pois consideramos que uma subordinação ao serviço onde atua tornará mais difícil um olhar e uma estratégia de intervenção dentro dos moldes aqui delineados.

Consideramos também importante destacar a necessidade de capacitação dos psicólogos, assim como a reflexão, o desenvolvimento de estratégias e o aperfeiçoamento constante para essa prática.

Samantha Mucci *

Psicóloga do SAPIS - Departamento de Psiquiatria, profissional de ligação no Serviço de Transplante de Fígado UNIFESP/EPM.

Fátima Lucchesi

Psicóloga do SAPIS - Departamento de Psiquiatria, profissional de ligação na Unidade de Terapia Intensiva Geral UNIFESP/EPM.

Tatiana Gottlieb Lerman

Psicóloga do SAPIS - Departamento de Psiquiatria, profissional de ligação na Enfermaria de Clínica Médica Feminina e Enfermaria de Hematologia e Transplante de Medula Óssea - UNIFESP/EPM.

Daniela Betinassi Parro-Pires

Psicóloga do SAPIS - Departamento de Psiquiatria, profissional de ligação na Enfermaria de Clínica Médica Masculina e da Unidade de Terapia Intensiva da Clínica Médica - UNIFESP/EPM.

Luciana Geocze

Psicóloga do SAPIS - Departamento de Psiquiatria, profissional de ligação na Enfermaria de Doenças Infecciosas e Parasitárias - UNIFESP/EPM.

Ligia Bruhn de Souza Aranha

Psicóloga do SAPIS - Departamento de Psiquiatria, profissional de ligação nas enfermarias cirúrgicas - UNIFESP/EPM.

Mario Alfredo De Marco

Professor associado, coordenador-geral do SAPIS- Departamento de Psiquiatria - UNIFESP/EPM.

* Endereço para envio de correspondência:

Rua Borges Lagoa, 570 - Departamento de Psiquiatria - Vila Clementino, CEP:04038-000 - São Paulo-SP

E-mail: sammucci@gmail.com

Recebido 03/07/2007 Reformulado 26/05/2008 Aprovado 07/06/2008 
Bader, A., Kremer, H., Erlich-Trungenberger, I., Rojas, R., Lohmann, M., \& Deobald, O. et al. (2006). An adherence typology: Coping, quality of life, and physical symptoms of people living with HIV/AIDS and their adherence to antiretroviral treatment. Medical Science Monitor, 12(12), 493-500.

Botega, N. J., Bio, M. R., Zomignani, M. A., Garcia Jr, C., \& Pereira, W. A. B. (1995). Transtornos do humor em enfermaria de clínica médica e validação de escala de medida (HAD) de ansiedade e depressão. Revista de Saúde Pública, 29(5), 359-363.

Botega, N. J., \& Smaira, S. I. (2006). Morbidade psiquiátrica no hospital geral. In N. J. Botega (Org.), Prática psiquiátrica no hospital geral: interconsulta e emergência (pp. 31-42). Porto Alegre: Artmed.

Brousseau, K. (2007). Psychosocial aspects of HIV treatment. JAMA: Journal of the American Medical Association, 297(2), 57-58.

Cavanaugh, S. A., Furlanetto, L. M., Creech, S. D., \& Powell, L. H. (2001). Medical illness, past depression and present depression: A predictive triad for in-hospital mortality. The American Journal of Psychiatry, 158(1), 43-48.

Cigognini, M. A., \& Furlanetto, L. M. (2006). Diagnosis and pharmacological treatment of depressive disorders in a general hospital. Revista Brasileira de Psiquiatria, 28(2), 97-103.

Craven, J. (1990). Psychiatric aspects of lung transplant: The Toronto Lung Transplant Group. Canadian Journal of Psychiatry, 35, 759-764.

Creed, F., Morgan, R., Fiddler, M., Marshall, S., Guthrie, E., \& House, A. (2002). Depression and anxiety impair healthrelated quality of life and are associated with increased costs in general medical inpatients. Psychosomatics, 43(4), 302-309.

De Marco, M. A. (2003). A face humana da medicina: do modelo biomédico ao modelo biopsicossocial (pp. 38-41). São Paulo: Casa do Psicólogo.

De Marco, M. A., Citero, V. A., \& Nogueira-Martins, L. A. (2007). Revisando conceitos: o papel da psiquiatria moderna no hospital geral e na atenção primária. Revista Brasileira de Psiquiatria, 29(2), 188-188.

Ferrari, H., \& Luchina, I. L. (1971). La interconsulta médicopsicológica en el marco hospitalario. Buenos Aires: Nueva Visión.
Frasur-Smith, N., Lesperance, F., \& Talajic, M. (1993). Depression following myocardial infarction impact on 6-month survival. JAMA: Journal of the American Medical Association, 270(15), 1819-1825.

Furlanetto, L. M., Del Moral, J. A. G., Gonçalves, A. H. B., Rodrigues, K., \& Jacomino, M. E. M. L. P. (2006). Diagnosticando depressão em pacientes internados com doenças hematológicas: Prevalência e sintomas associados. Jornal Brasileiro de Psiquiatria, 55(2), 96-101.

Lowry, M. R. (1979). Frequency of depressive disorder in patients entering home hemodialysis. Journal of Nervous and Mental Deseases, 167, 199-204.

Mari, J. J., \& Jorge, M. R. (1997). Transtornos psiquiátricos na clínica geral. Psychiatry On-line Brazil, 2(5), 1997. Recuperado em 25 de março de 2007, de http://www.polbr.med.br/ arquivo

Martin, A. G., Soler, R. S., \& Picart, P. A. (2003). Niveles de ansiedad y depresión en enfermos hospitalizados y su relación con la gravedad de la enfermedad. Medicina Clínica, 120(10), 370-375.

Quintana, A. M., Cecim, P. S., \& Henn, C. G. (2002). O preparo para lidar com a morte na formação do profissional de Medicina. Revista Brasileira de Educação Médica, 26(3), 204-210.

Rundell, J. R., \& Hal, R. C. W. (1997). Psychiatric characteristics of consecutively evaluated outpatient renal transplant candidates and comparisons with consultation-liaison inpatients. Psychosomatics, 38, 269-276.

Sarazua, E. F., Mas, M. B., \& San Gregório, M. A. P. (2005). AIDS and social exclusion: Personality features or adaptive behaviors? Spanish Journal of Psychology, 8(1), 45-55.

Schleifer, S. J., Macari-Hinson, M. N., Coyle, D. A., Slater, W. R., Kahn, M., \& Gorlin, R. et al. (1989). The nature and course of depression following myocardial infarction. Archives International Medicine, 149, 1785-1789.

Siegel, K., \& Schrimshaw, E. W. (2005). Stress, appraisal, and coping: A comparison of HIV-infected women in the preHAART and HAART eras. Journal of Psychosomatic Research, 58(3), 225-233.

Trzepacz, P. T., Brenner, R., \& Van Thiel, D. H. (1989). A psychiatric study of 247 liver transplantation candidates. Psychosomatics, $30,147-153$ 